\title{
An Evaluation Model for the Teaching Reform of the Physical Education Industry
}

\author{
Yuwei Sun and Miaomiao Jiang \\ Physical Education College of Harbin University of Commerce, Harbin 150028, China \\ Correspondence should be addressed to Miaomiao Jiang; 101866@hrbcu.edu.cn
}

Received 9 August 2021; Revised 28 September 2021; Accepted 22 October 2021; Published 17 November 2021

Academic Editor: Daqing Gong

Copyright (c) 2021 Yuwei Sun and Miaomiao Jiang. This is an open access article distributed under the Creative Commons Attribution License, which permits unrestricted use, distribution, and reproduction in any medium, provided the original work is properly cited.

In order to promote the development of sports industry teaching and accurately evaluate the teaching reform of sports industry, this paper constructs an evaluation model based on the teaching reform of sports industry. In addition, the k-means method is used to classify the teaching effect and simplify the data collection process, so as to improve the accuracy of teaching reform evaluation. The sample data come from the data released by the sports department and the government from 2017 to 2020 , as well as the actual survey. The 26 evaluation indexes were determined by expert survey, questionnaire interview, and relevant domestic literature. In addition, the Euclidean distance in K-means method is used to calculate the weight of each index, and the results of the evaluation model are analyzed. The results show that the evaluation accuracy of the sports industry teaching reform model proposed in this paper is $98.4 \%$ and the error is $1.3 \%$. The evaluation result is better than the previous ant colony model and is suitable for the evaluation of sports teaching reform.

\section{Introduction}

Sports industrialization teaching is an important measure to promote the development of national fitness. It is of great significance to improve physical quality and give full play to the social function of sports. The teaching contents and objectives of sports industry cannot meet the actual needs of teaching reform and affect the healthy development of sports industry, so the teaching of sports industry needs continuous reform. At present, the evaluation method of sports industrialization teaching reform is too subjective and lacks quantitative and qualitative analysis model. Therefore, building a teaching evaluation model of sports industry with high accuracy and small error is a problem that needs to be solved by the sports department at present. The results are shown as Figure 1.

The key to the teaching reform of sports industry is to give full play to its social function and socio-economic promotion. However, the teaching reform of sports industry lacks effective evaluation methods, especially comprehensive evaluation methods, which makes the teaching reform of sports industry unable to proceed smoothly. Based on the abovementioned background and reasons, this paper proposes an evaluation model based on improved ant colony algorithm and makes an experimental analysis on the teaching reform of sports industry to verify the effectiveness of this method.

\section{The Influence Factors of Physical Education Industry Teaching}

2.1. The Role of Physical Education Industry Teaching. The teaching reform of sports industry is a dynamic and complex social activity, involving many factors. Teaching reform includes not only national fitness and local economy but also national and government planning. Therefore, the teaching reform of sports industry needs accurate analysis and demonstration in order to achieve the expected goal. In addition, the teaching reform of sports industry also presents the characteristics of periodicity and randomness. Comprehensive analysis is needed to effectively identify the uncontrollable factors in the reform [1]. 


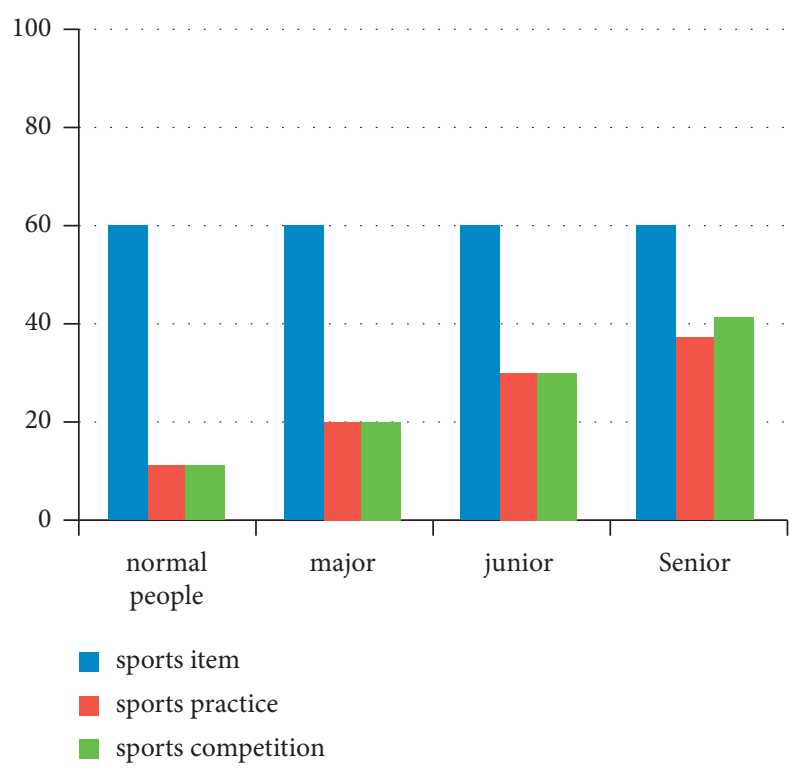

Figure 1: The results of sports in different grades.

2.2. The Effect of Teaching Reform of Physical Education Industry. A large number of studies show that regression analysis and other methods focus on controllable objective factors such as the study by Broo et al. [2], ignoring the correlation between controllable and uncontrollable factors. Therefore, there is a large deviation between the theoretical results and the actual results of the teaching reform of sports industry [3]. In addition, the teaching reform of sports industry is affected by many factors, and a single calculation method cannot meet the calculation requirements [4].

Based on the above analysis, this paper proposes an improved ant colony algorithm to analyze various factors of teaching reform in sports industry, extract the key indicators, and give weights to the indicators to form the final teaching reform model [5].The specific model calculation formula is expressed as follows:

$$
\left\{\begin{array}{l}
c_{i j}=x_{i}-\frac{\overline{x_{j}}}{\sqrt{s_{i j}}}, \\
\overline{x_{j}}=\sum_{i=1}^{n} x_{i j}, \\
s_{i j}=\sum_{i=1}^{n}\left(x_{i j}-\overline{x_{j}}\right)^{2},
\end{array}\right.
$$

where $i$ is the number of parameters, $j$ is the frequency of parameter values, $n$ is the number of samples, $c_{i j}$ is the amount after processing $x_{i j}, \bar{x}_{j}$ is the average value of $x_{i j}$, and $s_{i j}$ is the variance of $x_{i j}$.

\section{K-Means Classification}

In order to ensure the accuracy of teaching reform evaluation and simplify the data collection process, it is necessary to classify the teaching effect by $K$-means. This paper uses the $K$ - means classification of index division. In the process of data collection, there is no significant sudden change between the last test time $t_{i}$ and the next test time $t_{i+1}$ of each evaluation dimension, so there is a correlation between the evaluation dimensions of $t_{i}$ and $t_{i+1}$. Therefore, $t_{i+1}$ sports effect evaluation data come from TI's evaluation results, so as to realize the continuous evaluation of teaching effect. K-means classification is based on the sum of squared error clustering results, and its similarity judgment method is Euclidean distance. Among them, the standard K-means classification has the problems of large center ${ }_{P}$ deviation and inconsistent $K$-means of all kinds of samples, which affect the accuracy of $K$-means classification. In this paper, the concept of contour coefficient $S_{k}$ is introduced to limit the range of various types in $K$-means, so as to improve the accuracy of classification and the similarity of sample classification. The calculation formula of sample center is as follows:

$$
\left\{\begin{array}{l}
P(i)=\frac{p(i)-q(i)}{\max \{q(i), p(i)\}}, \\
\bar{P}=\frac{1}{n} \sum_{i=1}^{n} P(i) .
\end{array}\right.
$$

where $i$ is the sample ID, $q(i)$ is the distance from sample $i$ to other points in the cluster, $p(i)$ is the minimum distance from $i$ to other points in the noncluster, and $\overline{P(i)}$ is the average distance from the sample to the cluster. After obtaining the center of each sample, the total evaluation result of $P_{E}$ the whole dataset should be calculated. The calculation formula is as follows:

$$
P_{E}=\sqrt{\sum_{k=1}^{m} \omega_{k}\left(P_{k}-\min \left(P_{k}\right)\right)^{2} / k}, k=1,2 \cdots, m,
$$

where $\omega_{k}$ is the weight in the whole sample, the sum of which is $1, P_{k}$ is the center of different clustering samples, $\min \left(P_{k}\right)$ is the center of clustering $k$, and $P_{E}$ is the Euclidean distance between the average center and the minimum distance center of clustering $k$, representing the final evaluation result of sports effect. The teaching effect evaluation is arranged in ascending order of $P_{E}$, and the minimum value is selected as the initial cluster analysis value.

\section{Construction of Sports Teaching Effect Evaluation System}

4.1. Mathematical Description of the Improved Algorithm. The gradient descent logic in the standard ant colony algorithm will increase the number of local extremum and affect the accuracy of the results and the convergence speed of the calculation [6]. The K-means classification is integrated into the ant colony algorithm to construct the sports effect evaluation system. The contour coefficient $S_{k}$ can limit the gradient descent direction (positive and negative) in the process of ant colony algorithm, reduce the occurrence rate of local extremum, and improve the accuracy of calculation. $\omega_{k}$ can simplify the evaluation process of teaching reform in sports industry [7]. 
Suppose that the evaluation index vector of understanding sports in the sports teaching effect evaluation system is $x_{r}=\left(x_{1}, \ldots, x_{m}\right)^{T}$, the evaluation standard vector of subtle influence is $Y_{r}=\left(Y_{1}, \ldots, Y_{n}\right)^{T}$, the evaluation result vector of actual training is $O_{r}=\left(o_{1}, \ldots, o_{l}\right)^{T}$, and the expectation vector of teaching effect evaluation target is $D_{r}=\left(d_{1}, \ldots, d_{l}\right)^{T}$. Among them, the weight between understanding the sports evaluation index vector and the subtle influence is $w_{i k}$, contour coefficient is $S_{i k}$, the weight between the subtle influence and the actual training evaluation standard vector is $w_{k j}$, and contour coefficient is $S_{k j}$, and then the mathematical model of sports effect evaluation system is shown in the following formula:

$$
\left\{\begin{array}{l}
y_{j}=f\left[\overline{\sum_{i=1}^{m}\left(w_{i k} x_{i}+S_{i k}\right)},\right. \\
o_{k}=f\left[\sum_{j=1}^{p}\left(w_{k j} y_{i}+S_{k j}\right)\right] .
\end{array}\right.
$$

The output error between different layers $e=$ output vector $o$-expected vector $p$, and the calculation is shown in the following equation:

$$
\begin{aligned}
e & =\frac{1}{2}(D-O)^{2}=\frac{1}{2} \sum_{k=1}^{l}\left(d_{k}-f\left[\sum_{j=1}^{p}\left(w_{j k} y_{i}+b_{j k}\right)\right]\right)^{2} \\
& =\frac{1}{2} \sum_{k=1}^{l}\left(d_{k}-f\left[\sum_{j=1}^{p}\left(w_{j k} f\left[\sum_{i=1}^{m}\left(w_{i j} x_{i}+b_{i j}\right)\right]+b_{j k}\right)\right]\right)^{2} .
\end{aligned}
$$

Different input vectors get different output values, which makes a group of weights and thresholds of comprehensive construction effect, social function, and social economy realize internal representation. After repeated learning and national fitness, determine the network parameters with the minimum error and stop the analysis of national fitness. After the analysis of the effect of national fitness, the corresponding comprehensive evaluation is made for different objects.

Ant colony algorithm is divided into three layers: input layer, regulation layer, and output layer. When the input parameters are the same, different weights and thresholds will lead to different output results. In order to improve the accuracy of output results, reasonable weight setting should be carried out. In addition, in the process of teaching reform evaluation of sports industry, human subjective factors and objective factors of data collection will also lead to inaccurate output results. Therefore, the error adjustment function should be increased to reduce the influence of the above interference factors on the results.

\subsection{The Consistency Processing of Evaluation Data of Teaching Reform in Sports Industry}

4.2.1. Consistency of Social Function. Because the teaching evaluation data of sports industry comes from all aspects, including not only structured but also semistructured data, the inconsistency of data results will increase the computational complexity and prolong the calculation time of evaluation results. Therefore, it is necessary to standardize the data of teaching reform of sports industry. The specific calculation formula is shown as

$$
\iint d_{i}=\int \alpha \frac{\overline{\overline{\Delta d+\beta}}}{d_{\max }-d_{\min }+\beta},
$$

where $d_{i}$ is the teaching effect evaluation data, $\iint d_{i}$ is the result of $d_{i}$ normalization, and $d_{\max }$ and $d_{\min }$ are the maximum and minimum values of each evaluation index. Because the data projection is in the range of $(0,1)$, the values of each index approach and cannot reach 0 and 1 . Therefore, when $\iint d_{i}=d_{\max }, \alpha \approx 1$; when $\iint d_{i}=d_{\min }, \beta \approx 0$.

4.3. Error Analysis of Evaluation Results. The error of evaluation results is divided into mean square error $E_{m}$ and relative error percentage $E_{\mathrm{C}}$, both of which are used to evaluate the error from the qualitative and quantitative point of view [8], realizing the comprehensive evaluation of teaching effect evaluation. $E_{m}=$ estimated value-actual value, the overall result error of sports effect; $E_{\mathrm{C}}$ is the error percentage between samples in the index which reflects the error of the index, and the specific calculation is shown in the following formula:

$$
\left\{\begin{array}{l}
\lim _{x \longrightarrow \infty} E_{m}=\frac{\left[\sum_{i=1}^{n-1}\left(\widehat{d_{i}-\xi}\right)^{2}\right]}{n-1}, \\
\lim _{x \longrightarrow \infty} E_{C}=\frac{\xi-d_{i}}{d_{i}} \times 100 \% .
\end{array}\right.
$$

\section{The Empirical Analysis on the Evaluation of Teaching Reform in Physical Education Industry}

In order to verify the effect of improved ant colony algorithm on the evaluation of sports industry teaching reform, this paper collects 46 data of sports industry teaching reform and makes 200 iterative analyses on the results [9]. Each evaluation index is the average value, and the period from June 2017 to January 2021 is selected. At the same time, set the accuracy of the calculation result to 0.001 . The specific data results are shown in Table 1.

5.1. Sample K-Means Classification. Taking the national fitness method $x_{1}$, social function $x_{2}$, local economy $x_{3}$, and local policy $x_{4}$ as the initial indicators, combined with the data in Table 2, the effect of physical education industry teaching is studied, as shown in Table 2.

According to the above analysis, the similarity of the range of each input variable and the total input variable is high, and there is no significant difference. The K-means classification result is better. Determine the outline system of the whole sports effect evaluation system; determine the 
TABLe 1: Actual measurement results $(n=46)$.

\begin{tabular}{|c|c|c|c|c|}
\hline Test number & Fitness effect & Improvement of local economy & Result & Variance \\
\hline 1 & 10 & 24 & 6 & 0.81 \\
\hline 2 & 6 & 42 & 5 & 0.81 \\
\hline 3 & 5 & 35 & 5 & 1.89 \\
\hline 4 & 4 & 43 & 5 & 0.81 \\
\hline 5 & 5 & 45 & 6 & 1.08 \\
\hline 6 & 10 & 44 & 5 & 1.89 \\
\hline 7 & 5 & 25 & 5 & 1.35 \\
\hline 8 & 3 & 42 & 5 & 1.08 \\
\hline 9 & 10 & 30 & 6 & 1.08 \\
\hline 10 & 15 & 39 & 6 & 1.35 \\
\hline 11 & 2 & 26 & 6 & 1.89 \\
\hline 12 & 7 & 22 & 6 & 0.81 \\
\hline 13 & 10 & 36 & 5 & 1.89 \\
\hline 14 & 6 & 41 & 6 & 1.89 \\
\hline 15 & 9 & 41 & 6 & 1.89 \\
\hline 16 & 12 & 45 & 6 & 1.35 \\
\hline 17 & 14 & 32 & 5 & 0.81 \\
\hline 18 & 9 & 24 & 6 & 1.62 \\
\hline 19 & 4 & 30 & 5 & 1.08 \\
\hline 20 & 16 & 24 & 6 & 0.81 \\
\hline 21 & 9 & 25 & 5 & 1.62 \\
\hline 22 & 5 & 26 & 6 & 1.35 \\
\hline 23 & 16 & 36 & 6 & 1.35 \\
\hline 24 & 2 & 23 & 5 & 0.81 \\
\hline 25 & 14 & 38 & 6 & 1.89 \\
\hline 26 & 13 & 27 & 5 & 0.81 \\
\hline 27 & 13 & 29 & 5 & 1.62 \\
\hline 28 & 10 & 39 & 6 & 1.35 \\
\hline 29 & 2 & 37 & 5 & 1.89 \\
\hline 30 & 13 & 28 & 6 & 1.35 \\
\hline 31 & 3 & 29 & 6 & 1.89 \\
\hline 32 & 8 & 35 & 5 & 1.35 \\
\hline 33 & 8 & 33 & 6 & 1.35 \\
\hline 34 & 5 & 37 & 6 & 1.62 \\
\hline 35 & 9 & 24 & 5 & 1.08 \\
\hline 36 & 5 & 23 & 5 & 1.89 \\
\hline 37 & 16 & 26 & 6 & 1.35 \\
\hline 38 & 13 & 45 & 6 & 1.89 \\
\hline 39 & 2 & 45 & 6 & 1.35 \\
\hline 40 & 9 & 45 & 6 & 1.89 \\
\hline 41 & 4 & 45 & 5 & 0.81 \\
\hline 42 & 16 & 29 & 5 & 1.89 \\
\hline 43 & 8 & 26 & 5 & 1.08 \\
\hline 44 & 8 & 30 & 5 & 1.35 \\
\hline 45 & 10 & 37 & 6 & 1.62 \\
\hline 46 & 7 & 27 & 5 & 0.81 \\
\hline
\end{tabular}

TABLE 2: The sample classification results.

\begin{tabular}{lccccc}
\hline \multirow{2}{*}{ Category } & \multirow{2}{*}{ Number of samples } & \multicolumn{2}{c}{ Initial cluster center } & $x_{3}$ & $x_{4}$ \\
& & $x_{1}$ & $x_{2}$ & 102.2 & 135.7 \\
$x_{1}$ & 231 & 114.0 & 140.9 & 92.2 & 84.5 \\
$x_{2}$ & 102 & 79.6 & 85.4 & 134.4 & 7.5 \\
$x_{3}$ & 321 & 181.7 & 173.1 & 111.3 & 17.5 \\
$x_{4}$ & 162 & 148.4 & 124.5 & 54.6 & 171.7 \\
Total & 816 & 552.7 & 575.3 & 549.5 & 138.4 \\
\hline
\end{tabular}


classification number $k$ to get the optimal value. Firstly, 816 samples are normalized, $k$ is increased from 2 to 7 , and $S_{t}$ is the contour coefficients. The result is shown as Table 3.

When the number of clusters is $k=4$, the coefficient $S_{k}$ is the largest, which indicates that the clustering effect is the highest under the 4-equal partition. Therefore, this paper chooses $k=4$ as the initial number of clusters, and $P_{i}$ carries on the 4 -equal partition clustering. Then, $P_{E}$ are the teaching effect evaluation results of sports which are calculated and arranged in ascending order. The results show that the minimum value of $P_{E}$ is 0.786 , the maximum value is 4.232 , and the value range is $0.786-4.232$. Among them, $K$-means is an iterative clustering analysis algorithm. The step is to divide the data into $k$ groups, then randomly select $k$ objects as the initial clustering center and then calculate the clustering of each object and each seed. On the one hand, $K$ means method randomly selects sports industry projects to reduce the subjectivity of human evaluation and realize the quantitative analysis of the results; on the other hand, this method classifies the sports industry projects and realizes the qualitative analysis of the results. Therefore, K-means method has the comprehensive advantages of qualitative and quantitative analysis and is suitable for the needs of teaching reform and evaluation of sports industry.

\subsection{The Error Calculation of Evaluation Results of Teaching} Reform in Physical Education Industry. According to the calculation results in Figure 2, the error rate of the improved algorithm is significantly lower than that of the original algorithm, and the results of the improved algorithm are basically consistent with the actual results. Therefore, the accuracy of the improved algorithm is better, which is consistent with the actual results. The errors of sports effect are calculated by different iterations [10], and the evaluation results of sports effect under different target errors are calculated. The results expressed are shown in Figure 2.

As can be seen from Figure 2, with the increase of evaluation data of teaching reform in sports industry, the calculation accuracy of the improved ant colony algorithm is consistent with the actual situation and better than the standard ant colony algorithm. The results show that the improved ant colony algorithm is more stable under the same amount of data, mainly because $K$-mean classification can improve the convergence of the evaluation model.

5.3. The Calculation Accuracy Analysis of Teaching Evaluation of Physical Education Industry. In order to further verify the effectiveness of the improved algorithm, the calculation accuracy should be verified to ensure that it meets the evaluation requirements, i.e., $>95 \%$. Based on the effect of national fitness, social function, and local economy, this paper makes a comprehensive analysis on the function system of sports. The results show that the index of this method is between 0 and 60 , the social function is above $97 \%$, and the calculation time is controlled between 0 and 30 seconds. Therefore, the comprehensive results of sports effect, social function, and calculation time are in line with the requirements, and the
TABle 3: Contour coefficient $S_{k}$ under different $k$ values.

\begin{tabular}{lccc}
\hline$k$ value & $S_{k}$ coefficient & $k$ value & $S_{k}$ coefficient \\
\hline 2 & 1.622 & 5 & 1.892 \\
3 & 0.811 & 6 & 1.692 \\
4 & 1.892 & 7 & 1.687 \\
\hline
\end{tabular}

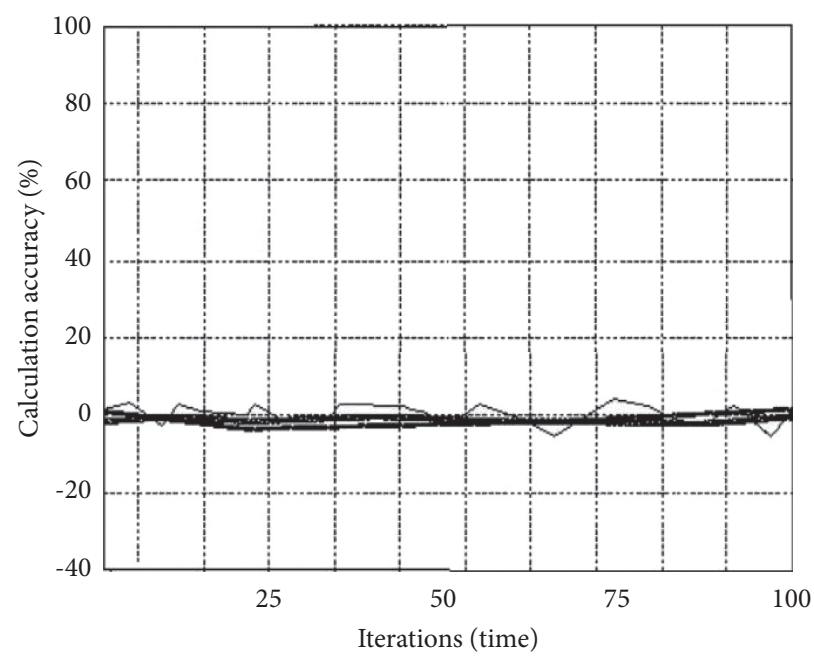

Improved ant colony algorithm
_ Ant colony algorithm results
_- - Actual statistical results

Figure 2: The error comparison of three methods.

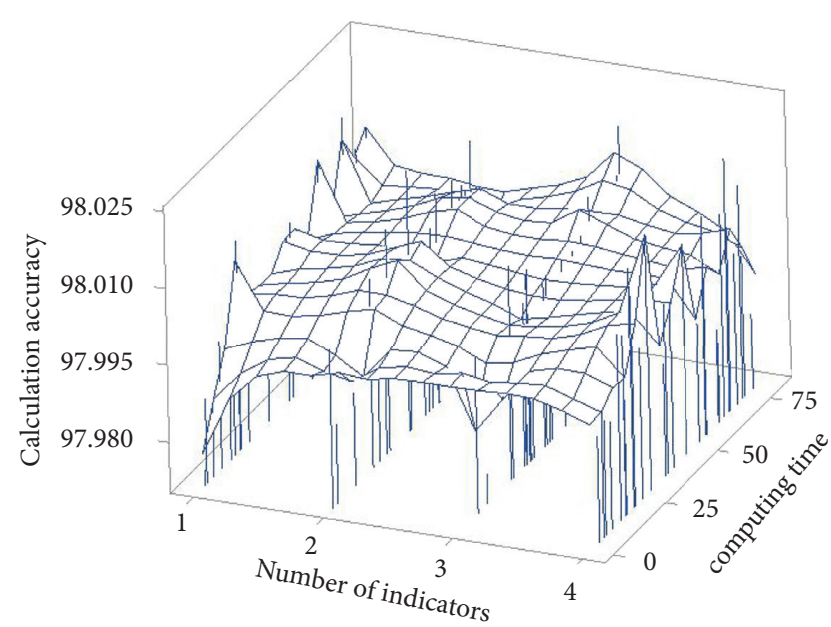

Figure 3: The comprehensive results of teaching effect evaluation of sports.

constructed quality action system can affect the actual movement. The results are shown as Figure 3.

The relationship between each index is also an important evaluation index of the algorithm and another important content to verify the effectiveness of the improved algorithm. In order to verify the effectiveness of the algorithm, the relationship between each index should be analyzed. By calculating the number of iterations from 0 to 1200 , the change 


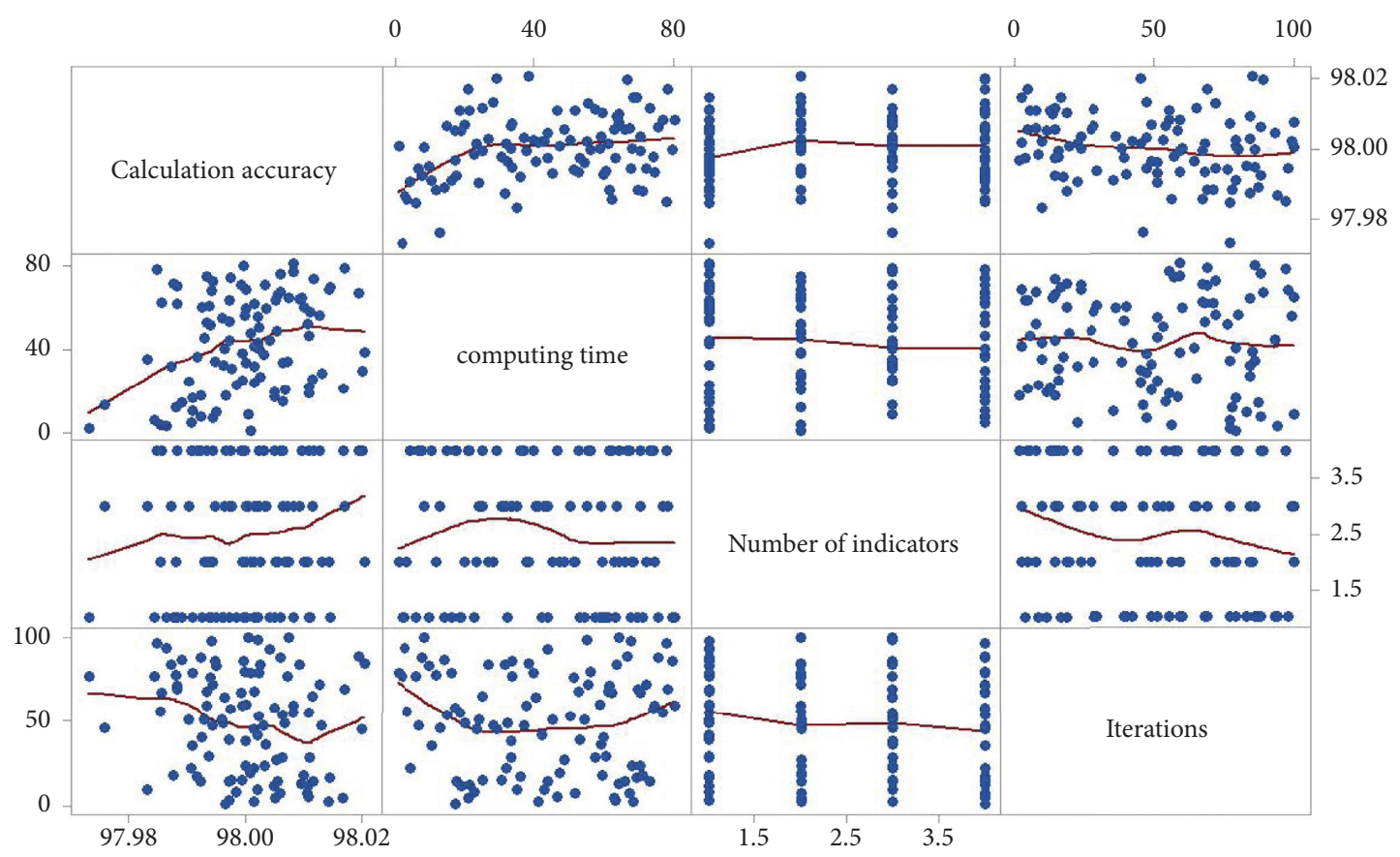

FIGURE 4: Relationship between iteration times and calculation accuracy, index number, and calculation.

values of the index number, calculation time, and calculation accuracy can be obtained. The result is shown as Figure 4.

It can be seen from the above analysis that with the increase of the number of iterations, the calculation accuracy of the teaching reform model of sports industry has not changed significantly, which is still between $97.5 \%$ and $98.5 \%$, maintained at about 30 , and the calculation time is maintained at about 17 seconds. Therefore, the overall effect of the sports industry teaching evaluation model based on the improved ant colony algorithm constructed in this paper is higher, which is better than the $90 \%$ accuracy of the previous genetic algorithm.

\section{Conclusions}

This paper analyzes the influence of physical education industry teaching on overall fitness effect [11], social function, and local economy. The collected sample data are normalized to reduce the impact of data attributes on the results [12]. Using MATLAB simulation analysis, the results show that, sports for the social function of national fitness [13], the role of local economy is more obvious. Through the convergence of the model, we can see that sports have a greater impact on national fitness [14]. At the same time, the function system constructed in this paper does not extend the calculation time with the increase of the number of iterations [15], which indicates that the effect of physical education industry teaching on national fitness is continuous [16]. Therefore, this paper believes that physical education industry teaching has a strong comprehensive role, can be used as an important part of the overall fitness, and promote the development of local economy, giving full play to the social function of national fitness.

\section{Data Availability}

The data used to support the results of this research are concealed and so cannot be provided.

\section{Conflicts of Interest}

The authors declare that they have no conflicts of interest.

\section{Acknowledgments}

This work was supported by the Heilongjiang Provincial Philosophy and Social Science Research Planning Project (18TYE554 and 17TYE351).

\section{References}

[1] T. Bopp and M. Stellefson, "Practical and ethical considerations for schools using social media to promote physical literacy in youth," International Journal of Environmental Research and Public Health, vol. 17, no. 3, pp. 201-202, 2020.

[2] D. G. Broo, U. Boman, and M. Torngren, "Cyber-physical systems research and education in 2030: scenarios and strategies," "Journal of Industrial Information Integration, vol. 21, no. 3, pp. 7-10, 2021.

[3] J. Cheng, "Evaluation of physical education teaching based on web embedded system and virtual reality," Microprocessors and Microsystems, vol. 32, no. 83, pp. 41-46, 2021.

[4] A. Cropley, "Creativity-focused technology education in the age of industry 4.0," Creativity Research Journal, vol. 32, no. 2, pp. 184-191, 2020.

[5] E. Enright, D. Kirk, and D. Macdonald, "Expertise, neoliberal governmentality and the outsourcing of health and physical education," Discourse: Studies in the Cultural Politics of Education, vol. 41, no. 2, pp. 206-222, 2020. 
[6] P. Holowka, "Reimagining the modern physical software engineering education and training environment," in Proceedings of the 32nd IEEE Conference on Software Engineering Education and Training (CSEE and T), vol. 61, no. 4, pp. 6-11, Germany, November 2020.

[7] N. Kamaludin and B. Mulyanti, "Cyber physical system in the industry," in Proceedings of the 3rd International Conference on Innovation in Engineering and Vocational Education, vol. 71, no. 8, pp. 102-104, Chennai, India, November 2019.

[8] V. Kazymyr, "Practical-oriented education in modeling and simulation for cyber-physical systems," in Proceedings of the 10th International Conference on Advanced Computer Information Technologies (ACIT), vol. 81, no. 5, pp. 61-69, Deggendorf, Germany, May 2020.

[9] P. Lahuta and M. Hudakova, "STREAMLINING professional qualification assessment process for private security employees," in Proceedings of the International Scientific Conference on the Impact of Industry 4.0 on Job Creation, vol. 61, no. 7, pp. 71-77, Trencianske Teplice, Slovakia, 2019.

[10] M. Malaga and Z. Ulrych, "Physical modelling of the Industry 4.0 concept," in Proceedings of the 35th International-BusinessInformation-Management-Association Conference (IBIMA), vol. 72, no. 8, pp. 61-62, Seville, Spain, April 2020.

[11] P. Marwedel, "Survey on education for cyber-physical systems," IEEE Design \& Test.vol. 37, no. 6, pp. 56-70, 2020.

[12] P. Mukucha, "The effects of business process outsourcing on the quality of catering services in tertiary education industry in Zimbabwe," Cogent Business \& Management, vol. 7, no. 1, pp. 32-34, 2020.

[13] R. S. Nagoyitsyn, "Interactive technologies in developing student's motivation in physical education and sport," International Journal of Applied Exercise Physiology, vol. 9, no. 6, pp. 78-85, 2020.

[14] T. W. Shen, "Development and evaluation of virtual reality induction electricity prevention education and training tools for construction industry," in Proceedings of the 7th IEEE International Conference On Consumer Electronics - Taiwan (ICCE-Taiwan), vol. 8, no. 7, pp. 6-11, Taiwan, September 2020.

[15] X. Y. Shi, "A didactic pedagogical approach toward sustainable architectural education through robotic tectonics," Sustainability, vol. 12, no. 5, pp. 7-10, 2020.

[16] M. Torngren, F. Asplund, and M. Magnusson, "The role of competence networks in the era of cyber-physical systems promoting knowledge sharing and knowledge exchange," IEEE Design and Test, vol. 37, no. 6, pp. 8-15, 2020. 